Article

\title{
A Mutational Analysis of Residues in Cholera Toxin A1 Necessary for Interaction with Its Substrate, the Stimulatory G Protein Gsa
}

\author{
Michael G. Jobling ${ }^{1}$, Lisa F. Gotow ${ }^{2}$, Zhijie Yang ${ }^{3}$ and Randall K. Holmes ${ }^{1, *}$
}

1 Department of Immunology and Microbiology, School of Medicine, University of Colorado Denver, 12800 E 19th Ave, Aurora, CO 80045, USA; E-Mail: michael.jobling@ucdenver.edu

2 Department of Biology, Metropolitan State University of Denver, P.O. Box 173362, CB 53, Denver, CO 80217, USA; E-Mail: lgotow@msudenver.edu

3 Atila Biosystems Inc., 740 Sierra Vista Ave, Unit E, Mountain View, CA 94043, USA; E-Mail: zhijie.yang@atilabiosystems.com

* Author to whom correspondence should be addressed; E-Mail: randall.holmes@ucdenver.edu; Tel.: +1-303-724-4223; Fax: +1-303-724-4226.

Academic Editor: Ken Teter

Received: 18 December 2014 / Accepted: 4 March 2015 / Published: 18 March 2015

\begin{abstract}
Pathogenesis of cholera diarrhea requires cholera toxin (CT)-mediated adenosine diphosphate (ADP)-ribosylation of stimulatory $\mathrm{G}$ protein $(\mathrm{Gs} \alpha)$ in enterocytes. $\mathrm{CT}$ is an AB5 toxin with an inactive CTA1 domain linked via CTA2 to a pentameric receptor-binding B subunit. Allosterically activated CTA1 fragment in complex with $\mathrm{NAD}^{+}$and GTP-bound ADP-ribosylation factor 6 (ARF6-GTP) differs conformationally from the CTA1 domain in holotoxin. A surface-exposed knob and a short $\alpha$-helix (formed, respectively, by rearranging "active-site" and "activation" loops in inactive CTA1) and an ADP ribosylating turn-turn (ARTT) motif, all located near the CTA1 catalytic site, were evaluated for possible roles in recognizing Gs $\alpha$. CT variants with one, two or three alanine substitutions at surface-exposed residues within these CTA1 motifs were tested for assembly into holotoxin and ADP-ribosylating activity against Gs $\alpha$ and diethylamino-(benzylidineamino)-guanidine (DEABAG), a small substrate predicted to fit into the CTA1 active site). Variants with single alanine substitutions at H55, R67, L71, S78, or D109 had nearly wild-type activity with DEABAG but significantly decreased activity with Gs $\alpha$, suggesting that the corresponding residues in native CTA1 participate in
\end{abstract}


recognizing Gs $\alpha$. As several variants with multiple substitutions at these positions retained partial activity against Gs $\alpha$, other residues in CTA1 likely also participate in recognizing Gs $\alpha$.

Keywords: cholera toxin; Gs alpha; ADP-ribosylation

\section{Introduction}

Cholera toxin (CT) is a major virulence factor produced by Vibrio cholerae, the causative agent of human cholera [1]. The action of CT on intestinal epithelial cells results in net fluid secretion and the massive watery diarrhea characteristic of cholera. CT is the prototype AB5 toxin, consisting of a single enzymatic A subunit non-covalently linked to a homopentameric B subunit [2]. The A and B polypeptides are made as pre-proteins, secreted into the periplasm, processed to mature forms, and assembled into catalytically inactive holotoxin that is exported across the outer membrane. CT binds to lipid raft-associated ganglioside GM1 receptors on enterocytes in the small intestine, is internalized by endocytosis, and traffics via the retrograde pathway through the Golgi to the endoplasmic reticulum (ER) [3]. CT is susceptible to proteolytic nicking of its A subunit within a disulfide-linked loop between the CTA1 and CTA2 domains, and subsequent reduction of that disulfide bond generates non-covalently-linked CTA1 and CTA2 fragments. Nicking can be accomplished either by Vibrio-produced proteases or proteases of enterocytes to which CT is exposed during uptake and internalization. Reduction occurs in the ER of intoxicated enterocytes, after which chaperone-facilitated dissociation from holotoxin initiates retrotranslocation of reduced CTA1 into the cytosol. Although the reduced CTA1 fragment has a basal level of catalytic activity, it is allosterically activated in the cytosol by binding to the GTP-bound forms of cellular co-factors called ADP-ribosylation factors (ARFs) [4,5]. Allosterically-activated CTA1 ADP-ribosylates and activates the alpha subunit of the stimulatory heterotrimeric G-protein (Gs $\alpha$ ) resulting sequentially in constitutive activation of adenylate cyclase, increased production of cAMP, activation of protein kinase A, phosphorylation and activation of the cystic fibrosis transmembrane conductance regulator chloride channel, and enhanced secretion of $\mathrm{Cl}^{-}$ions into the intestinal lumen [6]. These events, plus, cAMP-dependent inhibition of $\mathrm{Na}^{+}$-uptake [7], cause fluid loss into the intestine that presents clinically as watery diarrhea.

CTA1 undergoes several conformational changes during its transition from the catalytically latent CTA1 domain in holotoxin to the nicked, reduced and ARF-bound active CTA1 fragment that is presumed to ADP ribosylate Gs $\alpha$. The structure of a nicked but not reduced form of the related heat-labile enterotoxin LT-I showed no major conformational differences from un-nicked toxin [8]. The latent catalytic site within the CTA1 domain of holotoxin is occluded by an "active site" loop (consisting of residues 47-56) that is held in place by interactions with an "activation loop" (consisting of residues 25-36) that also interacts with CTA2 [9]. The structure of an enzymatically inactive R7K variant of LT provided early evidence that the corresponding activation loop of LT was disordered. Analysis of that structure generated a model whereby nicking and reduction of the holotoxin were proposed to initiate activation of the corresponding A1 domain by a three-step process, starting at the site of nicking and reduction, propagating to the active site, and involving: (1) increased flexibility of the long alpha helical segment of the A2 domain leading to disruption of its interaction with the "activation loop"; (2) increased flexibility of the "activation loop" leading to 
disruption of its interaction with the "active site" loop; and (3) increased flexibility of the "active site" loop enabling it be displaced and permit entry of the substrates NAD and the target arginine residue of Gs $\alpha$ into the active site [10]. Additional support for this model was provided by crystal structures of a CT variant with a Y30S substitution in the "activation" loop that permitted the holotoxin to exhibit intrinsic enzymatic activity without any requirement for proteolysis or reduction [9]. The "activation" loop in each of several crystal forms of this Y30S holotoxin variant was disordered, and the "active site" loop displayed varying degrees of order.

CTA1 has low intrinsic enzymatic activity in vitro, but interaction of CTA1 with any of several eukaryotic ARFs results in allosteric activation of its catalytic activity. A bacterial two-hybrid analysis of CTA1 and ARF6 showed that they form a tight interaction complex and identified residues in CTA1 that are required for binding to ARF6, thereby identifying a potential interaction interface [11]. Subsequent studies led to production in E. coli and purification of a complex containing a catalytically inactive CTA1 variant (with E110D and E112D substitutions) and ARF6-GTP, and comparisons of crystal structures of this complex (with or without bound NAD) [12] showed that residues 25-33 within the "activation loop" (residues 25-40) of CTA1 rearrange from an ordered coil in the catalytically latent holotoxin to a short amphipathic helix in the CTA1:ARF6-GTP complex (see Figure 1, Results and Discussion). Additional conformational changes that occur in CTA1 when it binds to ARF6-GTP include re-arranging residues 48-52 within the "active site" loop (residues 47-56) to form a knob near the active site and positioning the ADP ribosylating turn-turn (ARTT) motif (residues 104-110) near the active site. The ARTT motif is conserved among several ADP-ribosylating toxins (including pertussis toxin, CT, LT, diphtheria toxin, and Pseudomonas aeruginosa exotoxin A), and it participates in target protein recognition in some of them $[13,14]$. Taken together, these findings allowed us to predict several surface-exposed residues near the active site of CTA1 that might participate in binding and recognition of Gs $\alpha$ as an ADP ribosylation target. Nevertheless, Gs $\alpha$ alone is a poor substrate for CT, and under certain conditions CT can also ADP-ribosylate other G proteins including Gia [15-17]. Current biochemical and cell biological evidence

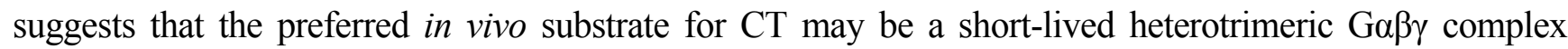
bound to an activated G protein coupled-receptor (GPCR) and with Gs $\alpha$ in a nucleotide-free state-i.e., after release of bound GDP but before G $\beta \gamma$ dissociates from Gs $\alpha$ and before Gs $\alpha$ binds GTP [18].

In vitro, $\mathrm{CT}$ can also ADP-ribosylate several small guanidino-group-containing artificial substrates that include agmatine [19] and diethylamino-(benzylidineamino)-guanidine (DEABAG) [20]. Structural and mutational studies identified several critically important residues of CTA1 or the related LTA1 that are required for enzymatic activity and NAD-substrate/ARF-co-factor binding [11,21-23], but the molecular interactions of CTA1 with Gs $\alpha$ are not well characterized. We hypothesized that ADP-ribosylation of a small artificial substrate like DEABAG would depend primarily on its interactions with ligands within the CTA1 active site, whereas ADP-ribosylation of the much larger protein substrate Gs $\alpha$ would likely require additional interactions with CTA1 ligands outside the active site. In this study, we introduced alanine substitutions into CT holotoxin at selected surface-exposed positions near the CTA1 active site, screened the purified, nicked and reduced holotoxin variants in vitro to identify ones that retained full or nearly-full ability to ADP ribosylate DEABAG but exhibited decreased ability to ADP ribosylate Gs $\alpha$, and thereby identified specific amino acid residues in CTA1 that likely participate in recognition and binding interactions with Gs $\alpha$. 


\section{Results and Discussion}

Figure 1 compares the conformation of a catalytically inactive CTA1E110D,E112D variant in complex with ARF6-GTP and NAD with the proenzyme form of wild-type CTA1 in native (un-nicked and un-reduced) CT. Based on the novel conformation of the allosterically-activated CTA1E110D,E112D fragment in complex with ARF6-GTP and NAD, we selected several surface-exposed residues near the active-site cleft to investigate as possible contributors to the interaction interface between CTA1 and Gs $\alpha$. The selected residues (see Figure 1) are as follows: R25 at the $N$-terminal end of the activation loop (residues 25-40); T50 and F52 (within the knob formed upon activation) plus H55 in the active-site loop (residues 47-56); R67 (not in a defined motif); L71, T75, I76, and S78 in a loop (residues 71-78) that may be comparable to the PN loop in the Ia fragment of the ADP-ribosylating iota toxin from Clostridium perfringens [24,25] (although lacking any amino-acid homology); and H107, D109, and E110 in the ARTT motif. It is noteworthy that the positions of most of these selected residues differ significantly between the proenzyme and allosterically-activated conformations of CTA1. Mutations encoding single or multiple alanine substitutions for these selected residues were introduced by site-directed mutagenesis into the $c t x A 1$-coding region of the inducible holotoxin-encoding plasmid clone pARCT5. Introduced mutations were confirmed by restriction digests of PCR amplicons of the ctxAl coding region to detect the novel restriction site(s) introduced with the alanine codon(s), followed by DNA sequencing to confirm that no unintended mutations were introduced in the cloned genes.

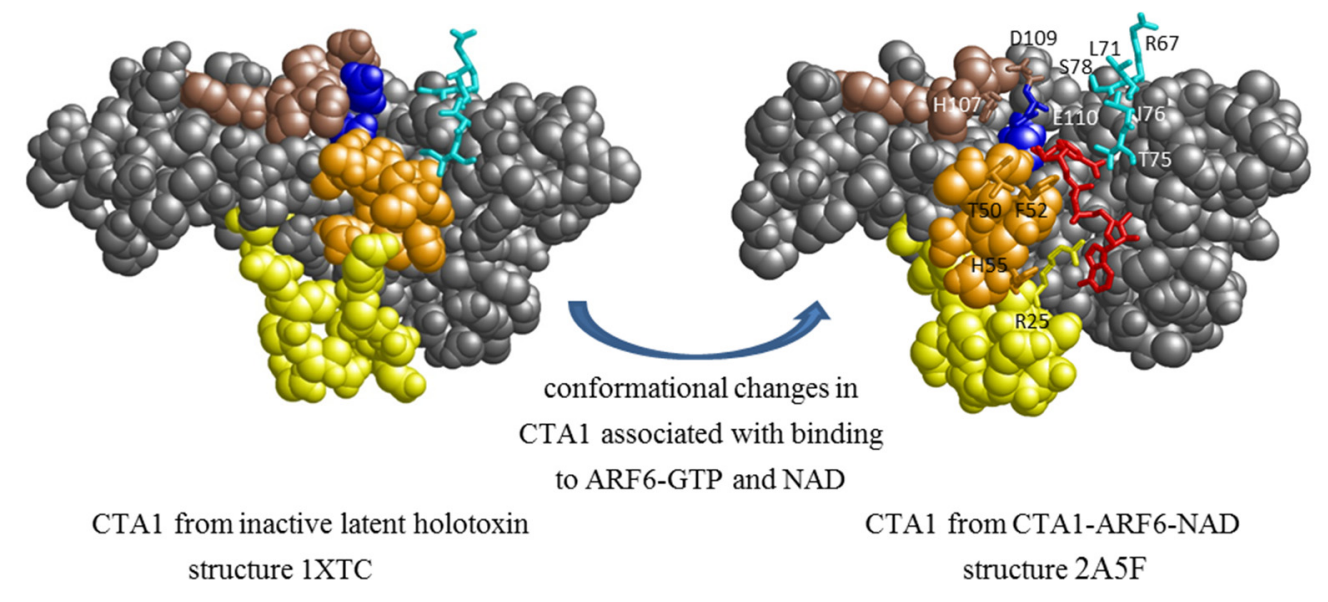

Figure 1. Conformational changes in cholera toxin A1 subunit CTA1 associated with binding to ADP-ribosylation factor 6 (ARF6)-GTP and NAD and predictions of candidate residues in CTA1 that may participate in Gs $\alpha$ binding. Space-filling projection of CTA1 $1_{\mathrm{wt}}$ from the crystal structures of latent holotoxin (1XTC, left) or CTA1E110D,E112D with $\mathrm{NAD}^{+}$and ARF6 (2A5F, right). Coloring and feature annotation is based on Figure 4B in reference [12]. ARF6 (not shown) binds to an interface on the rear of this projection. Residues 25-40 (activation loop) are yellow, residues 47-56 (active site loop, including knob in ARF6-bound form) are orange, residues 104-110 (ARTT motif) are brown, and active site residues 110 (stick) and 112 (space-fill) are blue. NAD is shown as sticks and is red. Each residue substituted by an alanine in this study is numbered, shown in stick format, colored cyan or as described above, and identified by the standard IUPAC one-letter code and position. 
Wild type (wt) and variant forms of CT holotoxin (listed below in the legend for Figure 2) were produced in E. coli, isolated from cellular extracts by Talon affinity chromatography, and further purified by ion-exchange chromatography. Holotoxins produced in E. coli have an intact CTA polypeptide that must be cleaved within the disulfide-linked loop joining the CTA1 and CTA2 domains and also reduced to produce the catalytically active CTA1 fragment. One sensitive measure of correct folding and assembly of variant toxins is their resistance to limited trypsin digestion. The CTA subunit of native toxin is nicked to give stable CTA1 and CTA2 fragments, while variants that are not folded correctly have A subunits that are rapidly and progressively degraded in the presence of limiting amounts of trypsin [22]. Therefore, we assessed the effects of limited trypsin digestion on the wt and variant forms of CT constructed for this study to see if any of the introduced alanine substitutions altered toxin stability. Each CT variant behaved comparably to wt CT and was nicked by trypsin to produce a stable A1 fragment, showing that these alanine substitutions did not measurably affect toxin assembly or resistance to limited trypsin digestion (Figure 2).

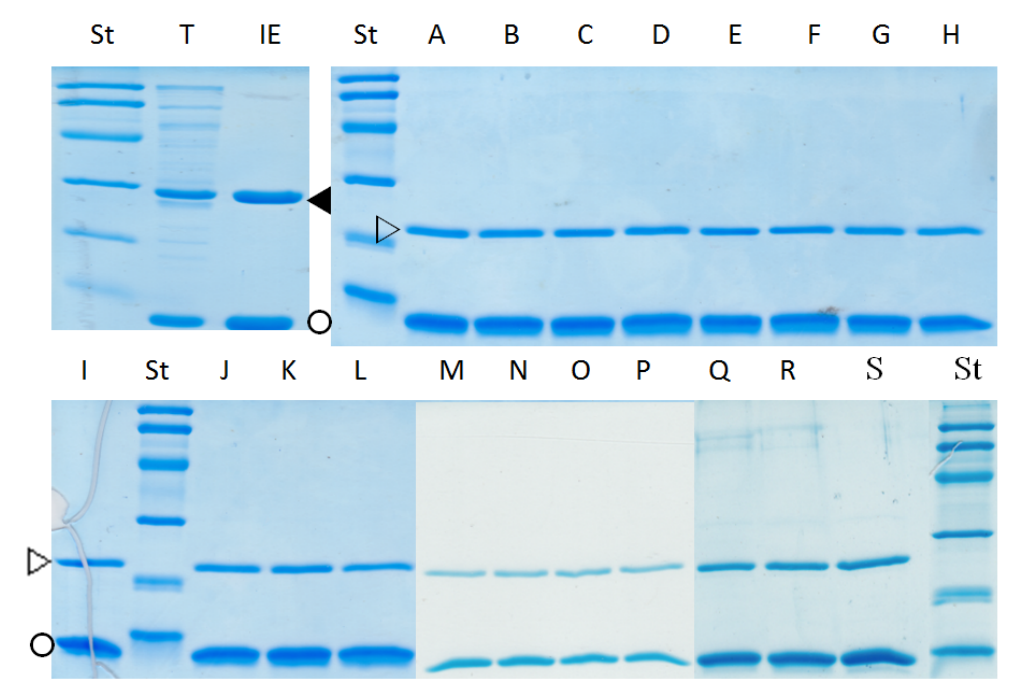

Figure 2. Purification and trypsin resistance of wt CT and CT variants with alanine substitutions in CTA1.SDS-PAGE analysis (15\% gels) of wt and variant holotoxins. All samples were boiled with 5\% $\beta$-ME prior to loading and electrophoresis at $200 \mathrm{~V}, 45$ mins, followed by colloidal staining with Coomassie Blue [26]. Top Left panel: St, protein standards; $\mathrm{T}$, partially purified wt CT after Talon purification; IE, purified wt CT after ion-exchange chromatography. Lanes $\mathrm{T}$ and IE each contain $2.5 \mu \mathrm{g}$ of un-nicked toxin. Top Right panel: analysis of $2.5 \mu \mathrm{g}$ samples of trypsin-treated wt and single- or double-alanine-substitution $\mathrm{CT}$ variants. St, protein standards; A, wt CT; B, CT-H55A; C, CT-R67A; D, CT-L71A; E, CT-S78A; F, CT-D109A; G, CT-H55A/L71A; H, CT-H55A/S78A. Bottom panel, analysis of $2.5 \mu \mathrm{g}$ samples of trypsin-treated double- or triple-alanine-substitution $\mathrm{CT}$ variants. I, CT-H55A/D109A; St, protein standards; J, CT-L71A/D109A; K, CT-S78A/D109A; L, CT-L71A/S78A; M, CT-R67A/S78A; N, CT-R67A/D109A; O, CT-H55A/L71A/S78A; P, CT-H55A/L71A/D109A; Q， CT-H55A/R67A; R, CT-H55A/R67A/L71A; S, CT-R67A/L71A; St, protein standards. Molecular masses: protein standards (top to bottom), 97, 66, 45, 31, 21.5 and $14 \mathrm{kDa}$; un-nicked CTA, $28 \mathrm{kDa}$ (filled triangle); CTA1, $21.5 \mathrm{kDa}$ (open triangle); CTB, $11.5 \mathrm{kDa}$ (circle); CTA2, $6.5 \mathrm{kDa}$ (does not stain). The figure is a composite of multiple gels that differ only in the degree of destaining. 
To analyze the biochemical and biological activities of these wt and variant CT holotoxins, we tested them in three assays: (1) ADP ribosyltransferase activity in vitro with the native substrate Gs $\alpha \beta \gamma$; (2) ADP ribosyltransferase activity in vitro with the artificial substrate DEABAG; and (3) toxicity for cultured Y1 adrenal cells (exhibited by increased intracellular cAMP). ADP-ribosylation of Gs $\alpha$ by CT was facilitated by the addition of G $\beta \gamma$ (Figure 3A), and G $\beta \gamma$ was included in all subsequent assays for ADP ribosylation of Gs $\alpha$. The apparent $K_{\mathrm{m}}$ for Gs $\alpha$ during ADP-ribosylation by wt CT was $1.95 \pm 0.23 \mu \mathrm{M}$ (Figure 3B). This $K_{\mathrm{m}}$ for Gs $\alpha$ is close to the calculated (low $\mu \mathrm{M}$ ) concentration of Gs $\alpha$ in rat myocytes (based on approximately $5 \times 10^{7}$ molecules of both short and long Gs $\alpha$ isoforms per cell [27] and an average cell volume of $34 \mathrm{pL}$ [28]). We then determined the activity of each of our CT variants for ADP-ribosylation of Gs $\alpha$ under identical conditions, and representative results are shown in Figure 3C.
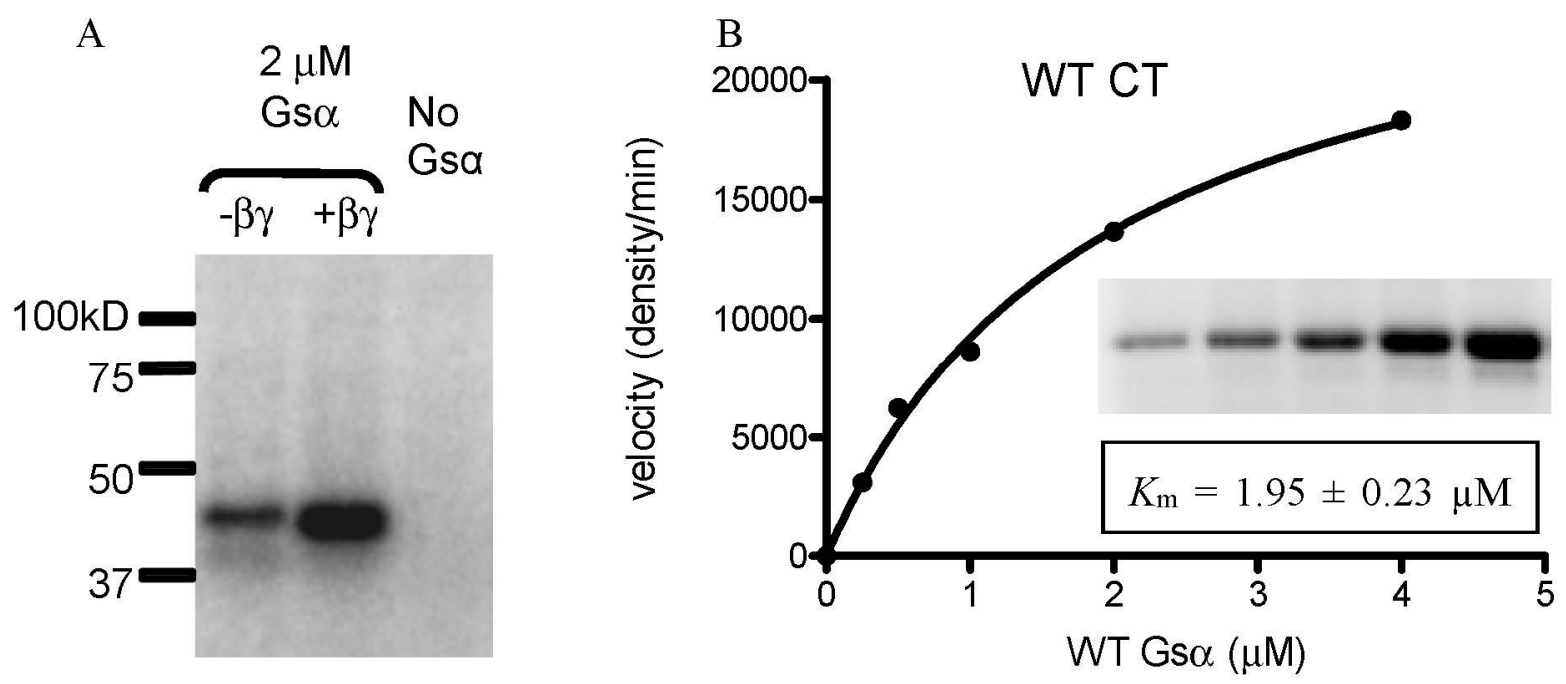

$\mathrm{C}$
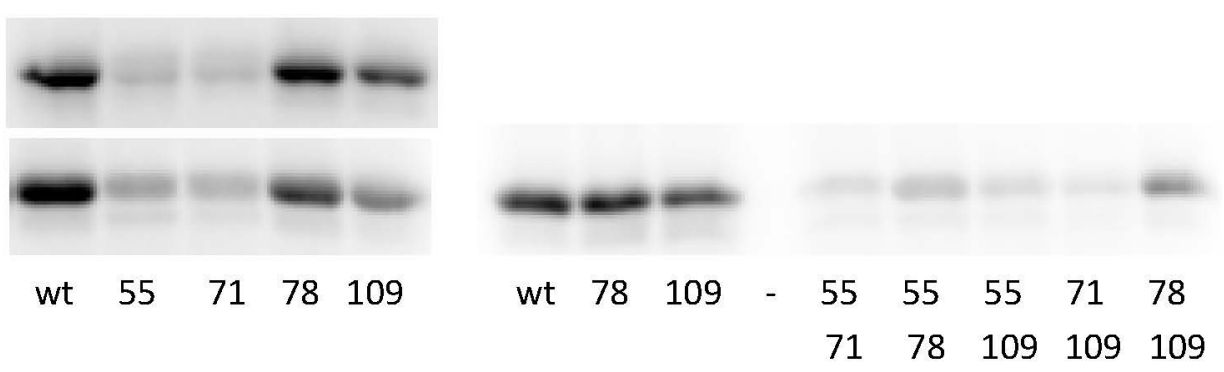

Figure 3. In vitro ADP-ribosylation of Gs $\alpha$ by nicked and reduced wt and variant forms of CT in the presence of ARF6-GTP and G $\beta \gamma$. Top left panel (A), G $\beta \gamma$ is required for efficient ADP-ribosylation of Gs $\alpha$ by wt CT. Top right panel (B), determination of apparent $K_{\mathrm{m}}$ for ADP-ribosylation of Gs $\alpha$ by wt CT. Lower left panel (C), reproducibility of Gs $\alpha$ ADP-ribosylation assay. Independent assays (upper and lower sections) were performed one week apart with (L to R) wt CT, CT-H55A, CT-L71A, CT-S78A, and CT-D109A. Lower right panel, ADP-ribosylation of Gs $\alpha$ by representative CT variants: (L to R) wt CT, CT-S78A, CT-D109A, empty lane, CT-H55A/L71A, CT-H55A/S78A, CT-H55A/D109A, CT-L71A/D109A, and CT-S78A/D109A. 
Quantitative ADP ribosylation assays catalyzed by wt CT and each of the CT variants were performed separately with Gs $\alpha$ and DEABAG as acceptor molecules, and for each set of assays the activity obtained with wt CT was set to 1.0 and the activity of each $\mathrm{CT}$ variant was normalized to the activity of wt CT (Figure 4). The CT-T75A variant did not differ significantly in activity from wt CT and was not considered further. The CT-I76A, CT-H107A and CT-E110A variants exhibited greatly decreased ADP-ribosyltransferase activity against both DEABAG and Gs $\alpha$.

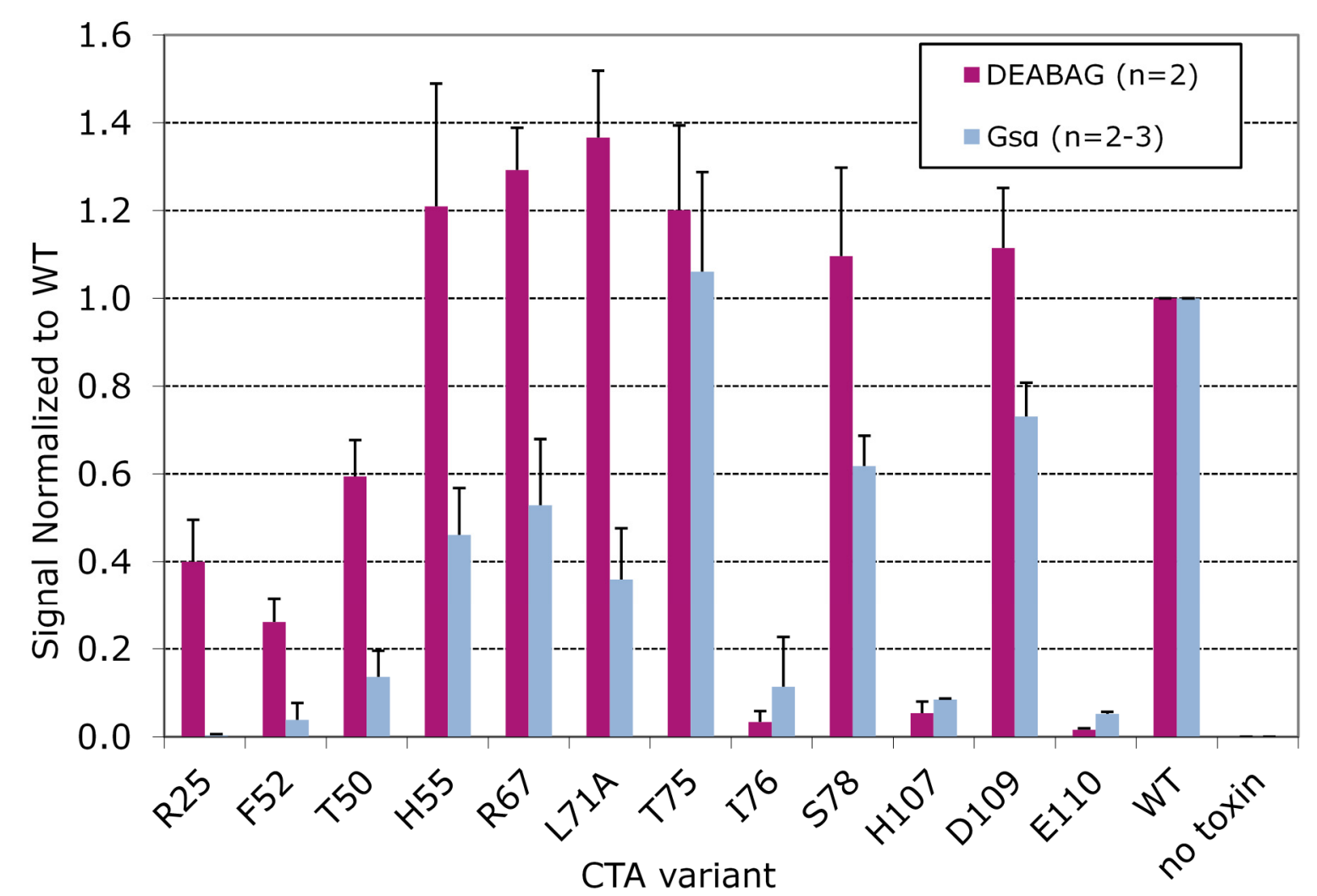

Figure 4. Relative activity of CTA variants in ADP-ribosylation assays with diethylamino-(benzylidineamino)-guanidine (DEABAG) or Gs $\alpha$.

Because the alanine substitutions in these three CT variants nearly abolished catalytic activity, we could not evaluate whether or not the I76, H107 and E110 residues contributed to recognition of Gs $\alpha$ by CTA1. The CT-R25A, CT-F52A, and CT-T50A variants exhibited partial activity against DEABAG but much less activity against Gs $\alpha$, suggesting that the wt residues at these positions contribute both to intrinsic catalytic activity of CTA1 and to recognition of Gs $\alpha$ by CTA1. Finally, the CT-H55A, CT-R67A, CT-L71A, CT-S78A, and CT-D109A variants exhibited full activity against DEABAG but substantially decreased activity against Gs $\alpha$, suggesting that the alanine substitutions at each of these positions decreased the ability of CTA1 to recognize Gs $\alpha$ without affecting the intrinsic catalytic activity of CTA1.

We also generated CT variants with two or three alanine substitutions distributed among positions $55,67,71,78$, or 109 in CTA1 to assess their cumulative effects on holotoxin assembly, susceptibility to degradation by trypsin, and catalytic activity against DEABAG and Gsa (Figure 5). 


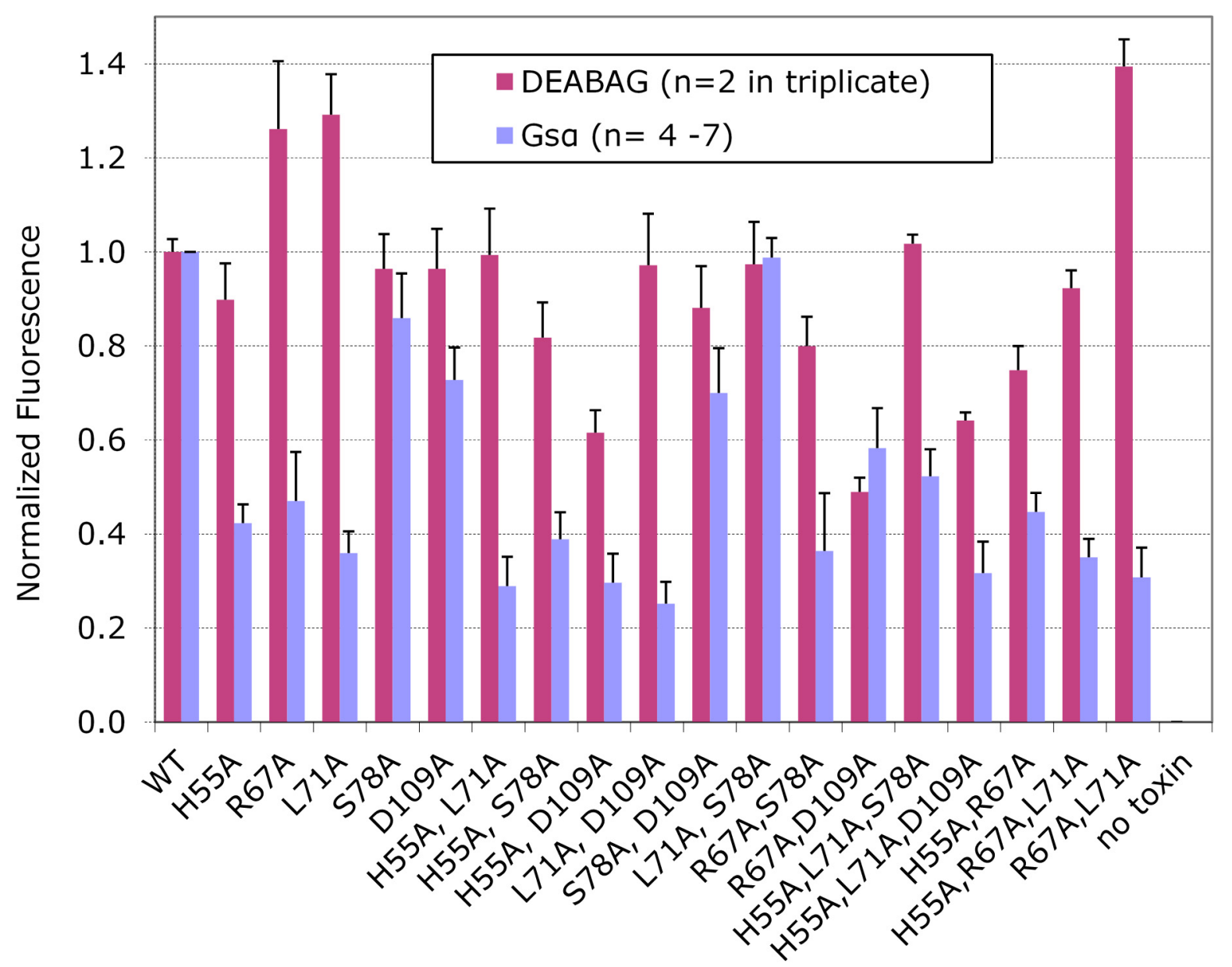

Figure 5. Effects of single-, double-, and triple-alanine-substitutions on the ratios of enzymatic activity for Gs $\alpha$ and DEABAG, normalized to the activities of wt CT.

We confirmed that these multiple substitutions did not affect toxin assembly or susceptibility to degradation by trypsin (see Figure 2, lanes G-I, L-N, and Q for representative data on double mutants; and lanes O-P and R-S for representative data on triple mutants). All of these doubly- and triply-alanine-substituted CT variants retained full or partial catalytic activity with DEABAG (Figure 5), and only the CT-H55A/D109A, R67A/D109A, and CT-H55A/L71A/D109A variants exhibited less than $75 \%$ of wt CT catalytic activity with DEABAG.

Most of the CT variants with multiple alanine substitutions retained ADP-ribosyltransferase for Gs $\alpha$ that was only slightly less than the activity of the least active of the corresponding single-alanine-substitution variants. In contrast, the CT-R67A/L71A double-substitution variant was slightly more active with DEABAG and slightly less active with Gs $\alpha$ than the CT-R67A and CT-L71A single-substitution variants, and it was substantially more active with DEABAG and less active with Gsa than wild-type CT. Furthermore, the CT-L71A/S78A double substitution variant had significantly greater activity with Gs $\alpha$ than the CT-L71A single substitution variant. We speculate that the diethylamino-benzylidine moiety of DEABAG interacts with CTA1 and that the R67A, L71A, or R67A plus L71A substitutions may increase CTA1 activity with DEABAG by modifying that interaction. It is also striking, that the CT variants with multiple alanine substitutions in CTA1 did not show dramatically greater losses of enzymatic activity with Gs $\alpha$ than the corresponding variants with single alanine substitutions. 
We determined apparent $K_{\mathrm{m}}$ 's for DEABAG and for Gs $\alpha$ in assays with wt CT and with each of the five CT variants that exhibited preferential loss of catalytic activity with Gs $\alpha$ and retention of full catalytic activity with DEABAG (Table 1). Each of these $5 \mathrm{CT}$ variants had an apparent $K_{\mathrm{m}}$ for DEABAG that was comparable or slightly lower than that of wt CT. In contrast, the CT-R67A and CT-L71A variants had apparent $K \mathrm{~m}$ 's for Gs $\alpha$ that were somewhat higher than that of wt CT. Furthermore, wt CT and the CT-H55A, CT-R67A and CT-L71A variants had comparable $K_{\mathrm{m}}$ 's for NAD (in the presence of $2 \mathrm{mM}$ DEABAG) that were also close to the reported intracellular concentration for NAD $(0.37 \mathrm{mM})$ in mouse erythrocytes [29].

Table 1. $K_{\mathrm{m}}$ determinations for CTA1 variants with Gs $\alpha$, DEABAG or NAD.

\begin{tabular}{cccc}
\hline CT Variant & Gs $\boldsymbol{\alpha}(\boldsymbol{\mu M})$ & DEABAG $(\mathbf{m M})$ & NAD $(\mathbf{m M})$ \\
\hline native & $1.66 \pm 0.88^{*}$ & $2.9 \pm 1.3$ & 0.354 \\
H55A & $1.8 \pm 0.4$ & $1.8 \pm 0.3$ & 0.334 \\
R67A & $4.5 \pm 1.2$ & $2.4 \pm 0.5$ & 0.382 \\
L71A & $2.5 \pm 0.5$ & $1.7 \pm 0.4$ & 0.299 \\
S78A & $1.5 \pm 0.3$ & $1.5 \pm 0.2$ & $\mathrm{ND}$ \\
D109A & $1.0 \pm 0.1$ & $3.2 \pm 0.5$ & $\mathrm{ND}$ \\
H55A + L71A & $8.6 \pm 4.0$ & $\mathrm{ND}$ & $\mathrm{ND}$ \\
H55A + S78A & $8.6 \pm 4.3$ & $\mathrm{ND}$ & $\mathrm{ND}$ \\
R67A + L71A & $5.0 \pm 1.0$ & $\mathrm{ND}$ & $\mathrm{ND}$ \\
H55A + R67A + L71A & $3.3 \pm 0.6$ & $\mathrm{ND}$ & $\mathrm{ND}$ \\
\hline
\end{tabular}

$* n=10$ (all others are $n=2-3 ; \mathrm{ND}$, not done).

The effects of wt CT on cultured Y1 adrenal cells are mediated by increases in the intracellular concentration of cAMP produced as a consequence of intoxication. We performed quantitative assays for intracellular cAMP in Y1 adrenal cells after exposing them to wt CT or to each of the five CT variants associated with full ADP ribosylating activity against DEABAG and selective loss of ADP ribosylating activity against Gs $\alpha$. Figure 6 compares the values for the in vitro ADP-ribosylation activity with DEABAG, the in vitro ADP-ribosylation activity with Gs $\alpha$, and the intracellular cAMP concentration for each of the CT variants, all normalized to values set to 1.0 for the corresponding results obtained with wt $\mathrm{CT}$. These results show that the in vivo cAMP concentrations in Y1 adrenal treated with each of these five CT variants correlate well with the ADP ribosyltransferase activities of the variants against Gs $\alpha$, but not against DEABAG, and support the conclusion that the residues replaced by alanine substitutions in these variants are directly involved in interactions of CTA1 with Gs $\alpha$. 


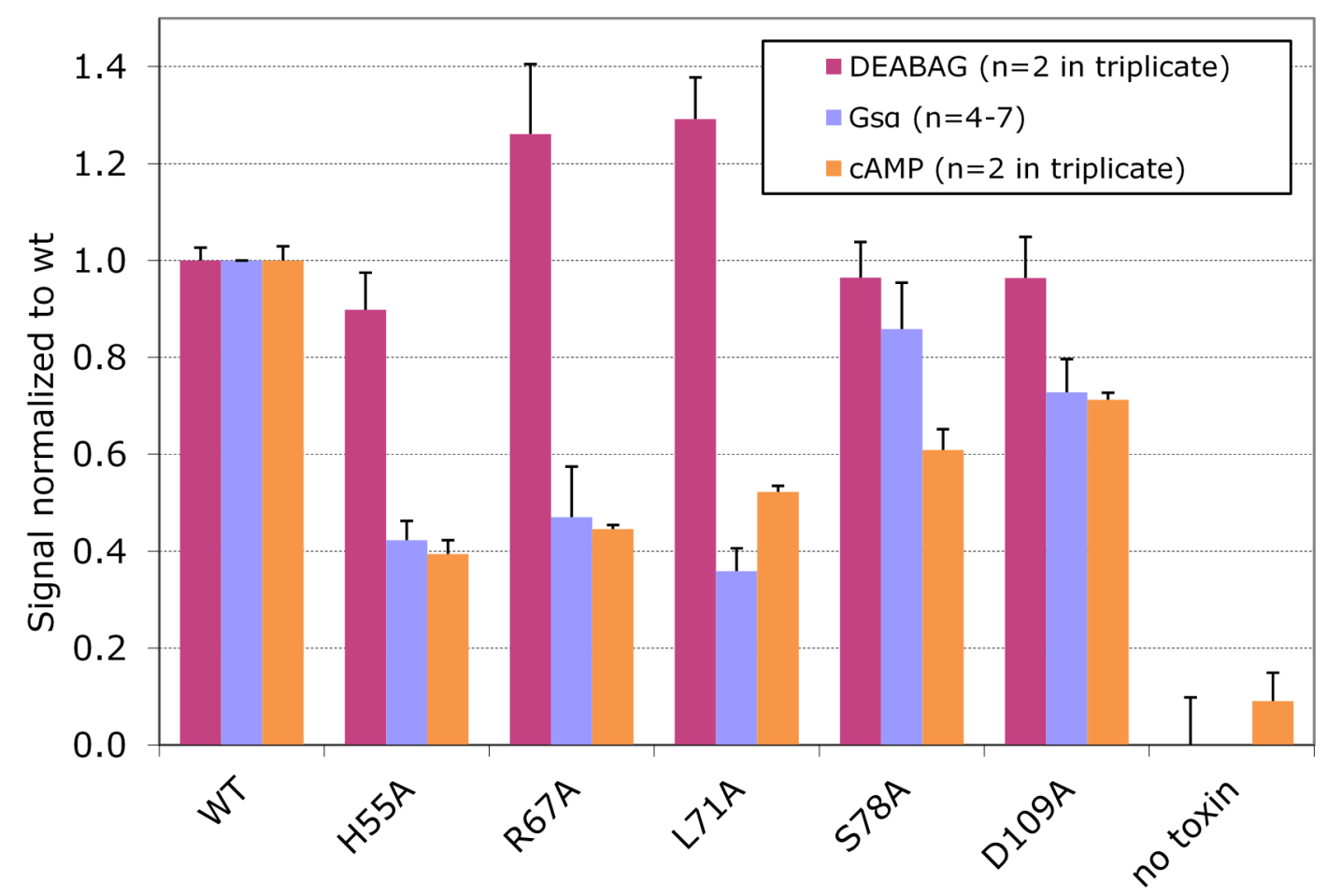

Figure 6. Normalized in vitro enzymatic activities with DEABAG or Gs $\alpha$ and normalized intracellular cAMP concentrations in intoxicated Y1 cells for selected CT variants $v s$. wt CT.

Toxin assays were done as described in experimental section, using equal amounts of wt or variant activated holotoxins ( $2 \mu \mathrm{g}$ for in vitro enzymatic assays and $1 \mathrm{ng}$ for in vivo cAMP assay). Calculated activities were then normalized to wt levels (wt CT =1.0) and plotted for each CT variant, showing SEM for replicates.

In this study, we identified five amino acids (H55, R67, L71, S78, and D109) located near but not in the active site of CTA1 that are likely involved in interactions with the Gs $\alpha$ substrate, based on the demonstration that single alanine substitutions at each of these positions decreased the ADP ribosylating activity of the corresponding CT variants for Gs $\alpha$ but not for the small artificial substrate DEABAG. We also identified three amino acids (R25, T50, and F52) that likely participate in interactions with Gs $\alpha$ substrate but also affect intrinsic catalytic activity, based on the demonstration that single alanine substitutions at each of these positions decreased the ADP ribosylating activity against both substrates, but caused substantially greater loss of activity for Gs $\alpha$ than for DEABAG. Because double and triple alanine substitutions did not cause substantially greater loss of ADP ribosylating activity with Gs $\alpha$ than single alanine substitutions, it seems likely that other residues in CTA1, yet to be identified, also make important contributions to binding interactions between CTA1 and Gs $\alpha$. If the binding interface between CTA1 and Gs $\alpha$ is large and complex, the consequences of any single alanine substitution for a residue in CTA1 that contributes to that interface may be small. It would be interesting in future studies to determine if more dramatic changes in substrate recognition specificity of CTA1 could be accomplished by substituting residues at the positions identified in the current study that would introduce larger side groups with various properties into the interface (instead of the methyl group provided by an alanine substitution), thereby creating potential steric hindrance as well as altering the local environment within the interface with respect to its hydrophobicity, 
hydrophilicity or charge. There is only one available structure for an arginine-specific ADP-ribosylating toxin in complex with its substrate, that of the Clostridium perfringens iota toxin Ia fragment with actin [24,25]. The Ia-actin interface involves 32 residues from each protein partner and covers $829-895 \AA^{2}$, or about $5 \%$ of the surface area of each protein. Furthermore, the interface involves five loops on the Ia fragment, only one of which has amino-acid homology in CTA1 (the ARTT loop), which include residues targeted in this study. Because actin and Gs $\alpha$ are not homologous, we cannot yet compare the relative contributions of amino acids at specific positions (other than the acceptor arginine residue) to the interactions of these substrates with their cognate ADP-ribosylating toxins.

We have shown here, and others have shown [30-32], that both in vitro and in vivo Gs $\alpha$ alone is a poor substrate for ADP-ribosylation, even in the presence of GTP-bound ARFs. Unlike the case for pertussis toxin, where peptides encompassing the last 10-20 amino acids of PT-sensitive alpha subunits (surrounding the target cysteine in Gi $\alpha$ ) are equally as effective substrates for PT-catalyzed ADP-ribosylation as is native Gia [33], CTA1 likely requires a very particular ternary complex that includes Gs $\alpha$ to enable ADP-ribosylation. Recent crystal structures of G protein complexes have given insight into why this is so. Early work with Gt (transducin) [18] and Gs $\alpha$ [34] produced data that were consistent with the natural substrate for cholera toxin being the activated GPCR-bound, nucleotide-free form of Gs $\alpha$. Gs $\alpha$ in the GDP bound (inactive) form has a high affinity for G $\beta \gamma$, and they form a stable heterotrimeric complex. In vivo, this complex is tightly bound to membrane-anchored quiescent GPCRs. Upon stimulation by receipt of an activation signal, the activated receptor induces a conformational change that results in opening of the interdomain interface in the $G$ protein heterotrimer with release of GDP, generating a nucleotide-free $\mathrm{G} \alpha(0)$ as part of the ternary complex of activated GPCR-G protein heterotrimer [35]. The structure of this activated complex has recently been determined [36,37] and reveals a remarkable conformational change in the nucelotide-free Gs $\alpha$ upon release of GDP, compared to its structure in the GPCR-coupled GDP-bound heterotrimer [38] or Gi $\alpha$ in the isolated heterotrimer [39]. This complex is short-lived, and the Gs $\alpha(0)$ rapidly binds GTP. Gs $\alpha(G T P)$ has dramatically reduced affinity for $G \beta \gamma$, and it quickly dissociates from the ternary complex and proceeds to activate downstream effectors, one of which in the case of Gs $\alpha$ is adenylate cyclase.

Our in vitro system for ADP-ribosylation of Gs $\alpha$ does not include a membrane-anchored GPCR or the upstream components required for its activation. Interestingly, it has been shown that ARF1 can also interact with Gs $\alpha$ and that the amino-terminus of ARF1 is required for CT-catalyzed ADP-ribosylation of Gs $\alpha$ [40], but not the small artificial substrate agmatine [40]. The presence of G $\beta \gamma$ and the other components in our system must be sufficient (but not necessarily optimal) to generate a form of Gs $\alpha$ that is ADP-ribosylated by CTA1. The availability of these new structures of the activated complexes should facilitate future studies on the interactions of CTA1 and Gs $\alpha$.

\section{Experimental Section}

\subsection{Generation of CT Variants by Site Directed Mutagenesis}

Alanine substitution mutations in the CTA1 coding region of a clone encoding an arabinose-inducible native CT operon (pARCT5) [22,41] were made by QuickChange mutagenesis using the manufacturer's protocol (Stratagene) (Table 2). Novel restriction sites were co-introduced 
along with the alanine substitution to enable tracking of changes. Double and triple substitutions were made by subcloning if suitable intervening restriction sites were available or alternatively by a second or third round of QuickChange mutagenesis. Each mutation was confirmed by DNA sequencing of the PCR amplified CTA1 coding region from the mutant plasmid clone.

\subsection{Protein Production and Purification}

Wild type CT holoxin and variants with alanine substitutions in the CTA1 domain were purified from extracts of cells from $400 \mathrm{~mL}$ of induced cultures. $50 \mathrm{~mL}$ overnight cultures in $\mathrm{TB}$ [42] grown at $30{ }^{\circ} \mathrm{C}$ were diluted into $400 \mathrm{~mL}$ of pre-warmed broth and grown to an $\mathrm{A} 600 \mathrm{~nm}$ of 3.5 and induced with $0.2 \% \mathrm{~L}$-arabinose for three hours. Induced cells were collected by centrifugation and resuspended in 1/20th volume of Talon buffer (50 mM Na phosphate, $\mathrm{pH} 8.0,0.3 \mathrm{M} \mathrm{NaCl}$ ) and extracted with $1 \%$ Elugent (Calbiochem) detergent. DNA was sheared by brief sonication, cell debris was removed by pelleting at $10,000 \times \mathrm{rpm}$ at $4{ }^{\circ} \mathrm{C}$, and the resulting extracts were purified by Talon affinity chromatography using the manufacturer's protocol, except that purified toxins were eluted with only $50 \mathrm{mM}$ imidazole. Holotoxins were purified further by two rounds of ion-exchange chromatography. Pooled fractions from the Talon elution were dialyzed into $50 \mathrm{mM}$ Tris $\mathrm{pH} 8.0$ (buffer A) and applied to an HS20 cation exchange column to remove free CTB penatamers. Holotoxin eluted in the flow-through, and the column was then washed with 5 column volumes $(\mathrm{CV})$ of buffer A. Free CTB pentamers were eluted with a $10 \mathrm{CV} 0 \%-100 \%$ gradient of buffer A to buffer B (buffer A with $1 \mathrm{M}$ $\mathrm{NaCl}$ ). The holotoxin-containing flow-through was applied directly to an HQ10 anion exchange column (to which holotoxin bound), and the column was subsequently washed with $5 \mathrm{CV}$ of buffer A after which holotoxin was eluted with a $10 \mathrm{CV} 0 \%-100 \%$ gradient of buffer A to buffer B. Fractions were analyzed by SDS-polyacrylamide gel electrophoresis and peak holotoxin fractions were pooled and dialyzed into buffer A for storage.

Table 2. Oligonucleotides used in this study.

\begin{tabular}{|c|c|c|}
\hline Name & $\begin{array}{c}\text { DNA Sequence } \\
\text { (forward Primer only, Changed Bases in Italics) }\end{array}$ & $\begin{array}{c}\text { Notes and Novel Restriction Site } \\
\text { Introduced (Underlined in Sequence) }\end{array}$ \\
\hline CTAXF & CGGGCAGATICTAGACCTCC & amplifies CTA1 for cloning with XbaI \\
\hline CTACR & CTCATCGATGATCTTGGAGCATTCCCACA & amplifies CTA1 for cloning with ClaI \\
\hline $\mathrm{R} 25 \mathrm{AF}$ & GGTCTTATGCCAGCTGGACAGAGTGAG & AluI \\
\hline $\mathrm{T} 50 \mathrm{AF}$ & AAGAGGAACTCAGGCCGGATTTGTTAGG & HaeIII \\
\hline F52AF & ACTCAGACGGGAGCTGTTAGGCACGATG & AluI \\
\hline H55AF & GGATTTGTTAGGGCCGATGATGGATATG & HaeIII \\
\hline R67AF & CTCAATTAGTTTGGCCAGTGCCCACTTAG & 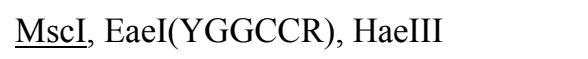 \\
\hline $\mathrm{L} 71 \mathrm{AF}$ & GTTTGAGAAGTGCCCACGCGGTGGGTCAAAC & BstUI \\
\hline $\mathrm{T} 75 \mathrm{AF}$ & CTTAGTGGGTCAAGCTATATTGTCTGGTC & AluI \\
\hline I76AF & GTGGGTCAAACTGCATTGTCTGGTCATTC & HpyCH4V \\
\hline S78AF & CAAACTATATTGGCCGGTCATTCTAC & HaeIII \\
\hline H107AF & GGCATACAGTCCTGCACCAGATGAACAAG & HруCH4V \\
\hline D109AF & CATACAGTCCTCATCCAGCTGAACAAGAAGTTTCTGC & PvuII, MspA1I (CMGCKG) \\
\hline E110AF & CCTCATCCAGATGCGCAAGAAGTTTCTGC & FspI, HhaI (GCGC) \\
\hline
\end{tabular}


Recombinant ARF6 was made from cytoplasmic extracts of IPTG-induced E. coli BL21(DE3) [pT7arf6] [11]. Cells were lysed by four cycles of freeze-thaw in TBS with $0.1 \%$ Triton-X100 and lysate viscosity was reduced by brief sonication or treatment with $10 \mu \mathrm{g} / \mathrm{mL}$ DNase I. Extracts were diluted 1:2 with $10 \mathrm{mM}$ Tris-EDTA and applied to a DEAE-sepharose column equilibrated with $20 \mathrm{mM}$ Tris $\mathrm{pH} 7.4,50 \mathrm{mM} \mathrm{NaCl}$. The majority of cellular proteins bound to the column, but rARF6 eluted in the flow-through and was then purified further over a second DEAE-sepharose column.

6-his-tagged-Gs $\alpha$ was made from soluble extracts of IPTG-induced cultures of E. coli BL21(DE3)[pUBS520, pQE60-his6Gs $\alpha$ ] as described by Yan and Tang [43], purified by Talon affinity and Q-sepharose ion exchange chromatography, eluted with $0.1 \mathrm{M}-0.5 \mathrm{M} \mathrm{NaCl}$ gradient in $20 \mathrm{mM}$ Tris pH 8.0, $5 \mathrm{mM} \beta$-mercapto-ethanol, $0.1 \mathrm{mM}$ PMSF, $1 \mathrm{mM} \mathrm{MgCl} 2$. Peak fractions were pooled, concentrated and buffer exchanged with 10,000 Da cutoff Amicon filters into $50 \mathrm{mM}$ Tris pH 8.0, $1 \mathrm{mM}$ EDTA, $1 \mathrm{mM}$ DTT and $10 \% v / v$ glycerol, aliquoted and stored at $-70{ }^{\circ} \mathrm{C}$. Functionality was tested in a GTP-binding assay using fluorescence of BODIPY-GTP [44].

Recombinant $\mathrm{G} \beta 1 \gamma 2$ was purified from $\mathrm{Sf} 9$ cells infected with Bacmid clones expressing his6-Gia1(G203A), G $\beta 1$ and G $\gamma 2$. Genes for the G-proteins were cloned into pFastBac1 and transposed into the bacmid backbone in DH10Bac cells with the Bac-to-Bac system from Invitrogen. Recombinant bacmids were confirmed by DNA sequencing, and transferred to the UCD tissue culture/Mab core facility to produce heterotrimeric G-protein complex by co-infection of Sf9 cells. The complex was purified from Sf 9 cell membranes by Talon affinity chromatography, and G $\beta 1 \gamma 2$ was eluted and purified from the complex as described [45].

\subsection{Enzymatic Assays}

Wild type and variant CT holotoxins were first activated by limited trypsin digestion. Samples of holotoxin were treated with $1 / 10^{\text {th }} \mathrm{w} / \mathrm{w}$ bovine trypsin and incubated at $37^{\circ} \mathrm{C}$ for $30 \mathrm{~min}$; trypsin was inactivated with the addition of $2 \times w / w$ soybean trypsin inhibitor; and the resulting samples were stable at $4{ }^{\circ} \mathrm{C}$. Enzymatic activity of variant holotoxins was measured quantitatively as previously described [46] using the small artificial substrate DEABAG at $2 \mathrm{mM}$, which is near its solubility limit [22]. All results were initially expressed as background-subtracted fluorescence units \pm SEM/assay ( $9988 \pm 50$ for wt); and the results for variant toxins were then normalized to values set to 1.0 for the results with wt CT. Reactions with Gs $\alpha$ as substrate were done in $60 \mu \mathrm{L}$ volumes of assay buffer $\left(50 \mathrm{mM}\right.$ potassium $\mathrm{PO}_{4}$ pH 7.5, $1 \mathrm{mM} \mathrm{MgCl}$, 3 mM DTT, $20 \mathrm{mM}$ thymidine, $100 \mu \mathrm{M}$ GTP, $10 \mu \mathrm{M}$ NAD) [47,48] with $2 \mu \mathrm{g}$ of activated wt or variant $\mathrm{CT}, 2 \mu \mathrm{M}$ rhis6Gs $\alpha, 0.5 \mu \mathrm{g}$ rG $\beta \gamma, 2 \mu \mathrm{g}$ rARF6 and $0.5-1 \mu \mathrm{L}{ }^{32} \mathrm{P}-\mathrm{NAD}$ $(5 \mu \mathrm{Ci} ; 800 \mathrm{Ci} / \mathrm{mmol} ; 5 \mathrm{mCi} / \mathrm{mL})$. Reactions were incubated for $1 \mathrm{~h}$ at $30{ }^{\circ} \mathrm{C}$, stopped by precipitation with TCA to also remove excess label, and washed with cold acetone. Pellets were resuspended in $30 \mu \mathrm{L}$ PBS and analyzed by SDS-PAGE. ${ }^{32} \mathrm{P}$-labeled Gs $\alpha$ at approximately $45 \mathrm{kDa}$ was quantitated on a Biorad Phosphorimager, expressed as background-subtracted counts $/ \mathrm{mm}^{2}$. Signals for equal amounts of individual CT variants was then normalized to $\mathrm{wt}=1.0$.

\subsection{In Vivo Toxicity Assays on Mouse Y1 Adrenal Cells}

Y1 cells (ATCC CCL-79) were cultured in RPMI-1640 medium with 10\% fetal calf serum in a humidified $5 \% \mathrm{CO}_{2}$ atmosphere. Cells were grown to semi-confluence in 96-well plates. Intracellular 
cAMP levels were quantitated in duplicate from acidic ethanol extracts of activated toxin-treated cells $\left(10 \mathrm{ng} / \mathrm{mL}, 100 \mu \mathrm{L} /\right.$ well for $2 \mathrm{~h}$ at $37^{\circ} \mathrm{C}$ ) using a Cayman Chemical cAMP EIA kit (581001) as described by the manufacturer; values were then normalized to wt ( $440 \pm 13 \mathrm{SEM} \mathrm{pmol/well;} \mathrm{no} \mathrm{toxin}$ (basal signal) $40 \pm 26 \mathrm{SEM} \mathrm{pmol/well).}$

\section{Conclusions}

By rational design, site-directed alanine mutagenesis, and phenotypic characterization of wt and mutant forms of CT, we identified several individual amino acid residues in CTA1 (H55, R67, L71, S78, and D109) that are likely involved in recognizing and binding the native substrate Gs $\alpha$. Single alanine substitutions for any of these surface-exposed residues had little or no effect on the ADP ribosylating activity of CTA1 for the small acceptor substrate DEABAG (which likely interacts within the active site cleft of CTA1), but they caused decreases in the ADP-ribosylating activity of CTA1 for the native acceptor substrate Gs $\alpha$ (a protein that likely binds to CTA1 by surface interactions during the process of ADP ribosylation of the target arginine-201 residue in Gs $\alpha$ ). In addition, CT variants with a single R25A, F52A or T50A substitution had moderately decreased activity with DEABAG but severely decreased activity with Gs $\alpha$, indicating that they likely participate both in catalytic activity and recognition of Gs $\alpha$.

\section{Acknowledgments}

This work was supported in part by NIH Grants R01AI031940 and R37AI014107 (to R.K.H.) from the National Institute of Allergy and Infectious Diseases, National Institutes of Health (http://www.nih.gov). The funders had no role in study design, data collection and analysis, decision to publish, or preparation of the manuscript. No other grants supported this study.

\section{Author Contributions}

Michael G. Jobling and Randall K. Holmes conceived the study and wrote the manuscript; all authors read and approved the final draft; Michael G. Jobling constructed the expression system clones; Lisa F. Gotow and Zhijie Yang constructed the CT variants and purified holotoxins; Lisa F. Gotow made the G protein constructs, purified the $\mathrm{G}$ proteins and performed the toxin assays.

\section{Conflicts of Interest}

The authors declare no conflict of interest.

\section{References}

1. Sack, D.A.; Sack, R.B.; Nair, G.B.; Siddique, A.K. Cholera. Lancet 2004, 363, 223-233.

2. Holmgren, J.; Sánchez, J. Cholera toxin-A foe \& a friend. Indian J. Med. Res. 2011, 133, 153-163.

3. Lencer, W.I. Retrograde transport of cholera toxin into the ER of host cells. Int. J. Med. Microbiol. 2004, 293, 491-494. 
4. Moss, J.; Vaughan, M. Activation of toxin ADP-ribosyltransferases by eukaryotic ADP-ribosylation factors. Mol. Cell. Biochem. 1999, 193, 153-157.

5. Banerjee, T.; Taylor, M.; Jobling, M.G.; Burress, H.; Yang, Z.; Serrano, A.; Holmes, R.K.; Tatulian, S.A.; Teter, K. ADP-ribosylation factor 6 acts as an allosteric activator for the folded but not disordered cholera toxin A1 polypeptide. Mol. Microbiol. 2014, 94, 898-912.

6. Kopic, S.; Geibel, J.P. Toxin mediated diarrhea in the 21st century: The pathophysiology of intestinal ion transport in the course of ETEC, $V$. cholerae and rotavirus infection. Toxins 2010, 2, 2132-2157.

7. Clarke, L.L.; Harline, M.C. CFTR is required for cAMP inhibition of intestinal $\mathrm{Na}^{+}$absorption in a cystic fibrosis mouse model. Am. J. Physiol. 1996, 270, G259-G267.

8. Merritt, E.A.; Pronk, S.E.; Sixma, T.K.; Kalk, K.H.; van Zanten, B.A.M.; Hol, W.G.J. Structure of partially-activated E. coli heat-labile enterotoxin (LT) at $2.6 \AA$ resolution. FEBS Lett. 1994, 337, $88-92$.

9. O’Neal, C.J.; Amaya, E.I.; Jobling, M.G.; Holmes, R.K.; Hol, W.G.J. Crystal structures of an intrinsically active cholera toxin mutant yield insight into the toxin activation mechanism. Biochemistry 2004, 43, 3772-3782.

10. Van den Akker, F.; Merritt, E.A.; Pizza, M.G.; Domenighini, M.; Rappuoli, R.; Hol, W.G.J. The Arg7Lys mutant of heat-labile enterotoxin exhibits great flexibility of active site loop 47-56 of the A subunit. Biochemistry 1995, 34, 10996-11004.

11. Jobling, M.G.; Holmes, R.K. Identification of motifs in cholera toxin A1 polypeptide that are required for its interaction with human ADP-ribosylation factor 6 in a bacterial two-hybrid system. Proc. Natl. Acad. Sci. USA 2000, 97, 14662-14667.

12. O’Neal, C.J.; Jobling, M.G.; Holmes, R.K.; Hol, W.G.J. Structural basis for the activation of cholera toxin by human ARF6-GTP. Science 2005, 309, 1093-1096.

13. Menetrey, J.; Flatau, G.; Boquet, P.; Menez, A.; Stura, E.A. Structural basis for the NAD-hydrolysis mechanism and the ARTT-loop plasticity of C3 exoenzymes. Protein Sci. 2008, 17, 878-886.

14. Han, S.; Tainer, J.A. The ARTT motif and a unified structural understanding of substrate recognition in ADP-ribosylating bacterial toxins and eukaryotic ADP-ribosyltransferases. Int. J. Med. Microbiol. 2002, 291, 523-529.

15. Klinz, F.-J.; Costa, T. Cholera toxin ADP-ribosylates the receptor-coupled form of pertussis toxin-sensitive G-proteins. Biochem. Biophys. Res. Commun. 1989, 165, 554-560.

16. Owens, J.R.; Frame, L.T.; Ui, M.; Cooper, D.M. Cholera toxin ADP-ribosylates the islet-activating protein substrate in adipocyte membranes and alters its function. J. Biol. Chem. 1985, 260, 15946-15952.

17. Iiri, T.; Tohkin, M.; Morishima, N.; Ohoka, Y.; Ui, M.; Katada, T. Chemotactic peptide receptor-supported ADP-ribosylation of a pertussis toxin substrate GTP-binding protein by cholera toxin in neutrophil-type HL-60 cells. J. Biol. Chem. 1989, 264, 21394-21400.

18. Bornancin, F.; Chabre, M. Cholera toxin ADP-ribosylates transducin only when it is bound to photoexcited rhodopsin and depleted of its nucleotide. FEBS Lett. 1991, 291, 273-276. 
19. Tsai, S.C.; Noda, M.; Adamik, R.; Moss, J.; Vaughan, M. Enhancement of choleragen ADP-ribosyltransferase activities by guanyl nucleotides and a $19-\mathrm{kDa}$ membrane protein. Proc. Natl. Acad. Sci. USA 1987, 84, 5139-5142.

20. Soman, G.; Narayanan, J.; Martin, B.L.; Graves, D.J. Use of substituted (Benzylidineamino) guanidines in the study of guanido group specific ADP-ribosyltransferase. Biochemistry 1986, 25, 4113-4119.

21. Burnette, W.N.; Mar, V.L.; Platler, B.W.; Schlotterbeck, J.D.; McGinley, M.D.; Stoney, K.S.; Rohde, M.F.; Kaslow, H.R. Site-specific mutagenesis of the catalytic subunit of cholera toxin: Substituting lysine for arginine 7 causes loss of activity. Infect. Immun. 1991, 59, 4266-4270.

22. Jobling, M.G.; Holmes, R.K. Biological and biochemical characterization of variant A subunits of cholera toxin constructed by site-directed mutagenesis. J. Bacteriol. 2001, 183, 4024-4032.

23. Pizza, M.; Domenighini, M.; Hol, W.; Gianelli, V.; Fontana, M.R.; Giuliani, M.M.; Magagnoli, C.; Peppoloni, S.; Manetti, R.; Rappuoli, R. Probing the structure-activity relationship of Escherichia coli LT-A by site-directed mutagenesis. Mol. Microbiol. 1994, 14, 51-60.

24. Tsuge, H.; Nagahama, M.; Oda, M.; Iwamoto, S.; Utsunomiya, H.; Marquez, V.E.; Katunuma, N.; Nishizawa, M.; Sakurai, J. Structural basis of actin recognition and arginine ADP-ribosylation by Clostridium perfringens I-toxin. Proc. Natl. Acad. Sci. USA 2008, 105, 7399-7404.

25. Tsurumura, T.; Tsumori, Y.; Qiu, H.; Oda, M.; Sakurai, J.; Nagahama, M.; Tsuge, H. Arginine ADP-ribosylation mechanism based on structural snapshots of iota-toxin and actin complex. Proc. Natl. Acad. Sci. USA 2013, 110, 4267-4272.

26. Kang, D.; Gho, Y.S.; Suh, M.; Kang, C. Highly sensitive and fast protein detection with coomassie brilliant blue in sodium dodecyl sulfate-polyacrylamide gel electrophoresis. Bull. Korean Chem. Soc. 2002, 23, 1511-1512.

27. Post, S.R.; Hilal-Dandan, R.; Urasawa, K.; Brunton, L.L.; Insel, P.A. Quantification of signalling components and amplification in the $\beta$-adrenergic-receptor-adenylate cyclase pathway in isolated adult rat ventricular myocytes. Biochem. J. 1995, 311, 75-80.

28. Satoh, H.; Delbridge, L.M.; Blatter, L.A.; Bers, D.M. Surface: volume relationship in cardiac myocytes studied with confocal microscopy and membrane capacitance measurements: Species-dependence and developmental effects. Biophys. J. 1996, 70, 1494-1504.

29. Yamada, K.; Hara, N.; Shibata, T.; Osago, H.; Tsuchiya, M. The simultaneous measurement of nicotinamide adenine dinucleotide and related compounds by liquid chromatography/electrospray ionization tandem mass spectrometry. Anal. Biochem. 2006, 352, 282-285.

30. Toyoshige, M.; Okuya, S.; Rebois, R.V. Choleragen catalyzes ADP-ribosylation of the stimulatory $\mathrm{G}$ protein heterotrimer but not its free a-subunit. Biochemistry 1994, 33, 4865-4871.

31. Graziano, M.P.; Casey, P.J.; Gilman, A.G. Expression of cDNAs for G proteins in Escherichia coli. Two forms of $\mathrm{G}_{\mathrm{s} \alpha}$ stimulate adenylate cyclase. J. Biol. Chem. 1987, 262, 11375-11381.

32. Schleifer, L.S.; Garrison, J.C.; Sternweis, P.C.; Northup, J.K.; Gilman, A.G. The regulatory component of adenylate cyclase from uncoupled S49 lymphoma cells differs in charge from the wild type protein. J. Biol. Chem. 1980, 255, 2641-2644.

33. Graf, R.; Codina, J.; Birnbaumer, L. Peptide inhibitors of ADP-ribosylation by pertussis toxin are substrates with affinities comparable to those of the trimeric GTP-binding proteins. Mol. Pharmacol. 1992, 42, 760-764. 
34. Bornancin, F.; Audigier, Y.; Chabre, M. ADP-ribosylation of Gs by cholera toxin is potentiated by agonist activation of $\beta$-adrenergic receptors in the absence of GTP. J. Biol. Chem. 1993, 268, 17026-17029.

35. Van Eps, N.; Preininger, A.M.; Alexander, N.; Kaya, A.I.; Meier, S.; Meiler, J.; Hamm, H.E.; Hubbell, W.L. Interaction of a $\mathrm{G}$ protein with an activated receptor opens the interdomain interface in the $\alpha$ subunit. Proc. Natl. Acad. Sci. USA 2011, 108, 9420-9424.

36. Chung, K.Y.; Rasmussen, S.G.F.; Liu, T.; Li, S.; DeVree, B.T.; Chae, P.S.; Calinski, D.; Kobilka, B.K.; Woods, V.L.; Sunahara, R.K. Conformational changes in the G protein Gs induced by the $\mathrm{b}_{2}$ adrenergic receptor. Nature 2011, 477, 611-615.

37. Westfield, G.H.; Rasmussen, S.G.F.; Su, M.; Dutta, S.; DeVree, B.T.; Chung, K.Y.; Calinski, D.; Velez-Ruiz, G.; Oleskie, A.N.; Pardon, E.; et al. Structural flexibility of the Gas $\alpha$-helical domain in the $\beta_{2}$-adrenoceptor Gs complex. Proc. Natl. Acad. Sci. USA 2011, 108, 16086-16091.

38. Rasmussen, S.G.F.; DeVree, B.T.; Zou, Y.; Kruse, A.C.; Chung, K.Y.; Kobilka, T.S.; Thian, F.S.; Chae, P.S.; Pardon, E.; Calinski, D.; et al. Crystal structure of the $\mathrm{b}_{2}$ adrenergic receptor-Gs protein complex. Nature 2011, 477, 549-555.

39. Wall, M.A.; Coleman, D.E.; Lee, E.; Iniguez-Lluhi, J.A.; Posner, B.A.; Gilman, A.G.; Sprang, S.R. The structure of the $G$ protein heterotrimer $\mathrm{G}_{\mathrm{ia} 1} \mathrm{~b}_{1} \mathrm{~g}_{2}$. Cell 1995, 83, 1047-1058.

40. Randazzo, P.A.; Terui, T.; Sturch, S.; Kahn, R.A. The amino terminus of ADP-ribosylation factor (ARF) 1 is essential for interaction with $\mathrm{G}_{\mathrm{s}}$ and ARF GTPase-activating protein. J. Biol. Chem. 1994, 269, 29490-29494.

41. Jobling, M.G.; Holmes, R.K. Characterization of cholera toxin variants with selected amino acid substitutions or insertions in the enzymatic domain of subunit A. Int. J. Med. Microbiol. 2000, 290, A48.

42. Tartof, K.D.; Hobbs, C.A. Improved media for growing plasmid and cosmid clones. Focus 1987, 9, 12.

43. Yan, S.-Z.; Tang, W.-J. Expression of alpha subunit of $\mathrm{G}_{\mathrm{s}}$ in Escherichia coli. Methods Enzymol. 2002, 344, 171-175.

44. McEwen, D.P.; Gee, K.R.; Kang, H.C.; Neubig, R.R. Fluorescent BODIPY-GTP analogs: Real-time measurement of nucleotide binding to G proteins. Anal. Biochem. 2001, 291, 109-117.

45. Ueda, N.; Iniguez-Lluhi, J.A.; Lee, E.; Smrcka, A.V.; Robishaw, J.D.; Gilman, A.G. G protein $\beta \gamma$ subunits. Simplified purification and properties of novel isoforms. J. Biol. Chem. 1994, 269, 4388-4395.

46. Narayanan, J.; Hartman, P.A.; Graves, D.J. Assay of heat-labile enterotoxins by their ADP-ribosyltransferase activities. J. Clin. Microbiol. 1989, 27, 2414-2419.

47. Kahn, R.A.; Gilman, A.G. ADP-ribosylation of Gs promotes the dissociation of its a and $b$ subunits. J. Biol. Chem. 1984, 259, 6235-6240.

48. Gill, D.M.; Meren, R. ADP-ribosylation of membrane proteins catalyzed by cholera toxin: Basis of the activation of adenylate cyclase. Proc. Natl. Acad. Sci. USA 1978, 75, 3050-3054.

(C) 2015 by the authors; licensee MDPI, Basel, Switzerland. This article is an open access article distributed under the terms and conditions of the Creative Commons Attribution license (http://creativecommons.org/licenses/by/4.0/). 